\title{
Expanding intestinal segment using osmotic hydrogel: An in vivo study
}

\author{
Riccardo Coletta, ${ }^{1,2,4}$ Claudio Olivieri, ${ }^{3}$ Giorgio Persano, ${ }^{4}$ Valeria Solari, ${ }^{2}$ Alessandro Inserra, ${ }^{3}$ \\ Antonino Morabito $2,3,4,5$ \\ ${ }^{1}$ Institute of Human Development, Faculty of Medical and Human Sciences, University of Manchester, Manchester, UK \\ ${ }^{2}$ Paediatric Autologous Bowel Reconstruction and Rehabilitation Unit, Department of Paediatric Surgery, Royal Manchester \\ Children's Hospital, Manchester, UK \\ ${ }^{3}$ Division of General and Thoracic Surgery, Bambino Gesù Children Hospital, Rome, Italy \\ ${ }^{4}$ Department of Pediatric Surgery, Meyer Children's Hospital, University of Florence, Florence, Italy \\ ${ }^{5}$ Institute of Inflammation and Repair, Faculty of Medical and Human Sciences, University of Manchester, Manchester, UK
}

Received 12 February 2018; revised 19 May 2018; accepted 12 August 2018

Published online 00 Month 2018 in Wiley Online Library (wileyonlinelibrary.com). DOI: 10.1002/jbm.b.34224

\begin{abstract}
Intestinal circumferential expansion is essential for bowel lengthening in patients with Short Bowel Syndrome. We hypothesized use of an endoluminal osmotic hydrogel expander (EOHE) as a novel approach for intestinal expansion. An EOHE was introduced into an isolated intestinal segment of New Zealand rabbits, with a similar segment created as a control. After 4weeks, the segments were retrieved for analysis. Weight, inflammatory markers and fluoroscopy data was recorded weekly. EOHE allowed successful expansion of intestinal segments from $4.68 \pm 0.35$ to $9.79 \pm 0.35 \mathrm{~cm}(p=0.01)$. Increase in intestinal length was $167.8 \pm 35.21 \%$ in segments with EOHE vs. $23.03 \pm 4.2 \%$ in the control group $(p<0.01)$. A significant intestinal dilatation $(214.4 \pm 1.58$ vs. $34.59 \pm 1.23 \%$,
\end{abstract}

$p<0.01$ ) was demonstrated. Hematoxylin and eosin stain revealed conservation of intestinal architecture with muscle hypertrophy and flattening of the epithelium possibly due to compression. No reduction of rabbit weight, inflammatory markers or liver damage was described. EOHE appears to produce safe intestinal expansion, achieving increased length and dilatation suitable for lengthening procedure. This approach may allow development of similar techniques to expand bowel in short bowel patients. (c) 2018 Wiley Periodicals, Inc. J Biomed Mater Res B Part B: Appl Biomater, 00B: 000-000, 2018.

Key Words: osmotic hydrogel device, distraction enterogenesis, short bowel syndrome, pediatric, intestine

How to cite this article: Coletta R, Olivieri C, Persano G, Solari V, Inserra A, Morabito A. 2018. Expanding intestinal segment using osmotic hydrogel: An in vivo study. J Biomed Mater Res B Part B. 2018:00:00:1-6.

\section{INTRODUCTION}

Short Bowel Syndrome (SBS) is a condition secondary to extensive bowel surgery in which the remnant intestine is not able to absorb sufficient nutrients for normal growth. ${ }^{1}$

The natural intestinal adaptation process after extensive bowel resection is able to generate dilatation of an intestinal segment allowing intestinal rehabilitative surgeons to perform autologous gastrointestinal procedures. ${ }^{2}$ Unfortunately, in some patients intestinal adaptation does not produce dilatation and therefore lengthening and tailoring surgeries are not feasible. ${ }^{3}$ In this scenario, the possibility to reach enteral autonomy is very limited ${ }^{4}$ and total parenteral nutrition (TPN) is essential for natural development. ${ }^{5,6}$ This exposes the patients to the severe TPN complications of intestinal failure and liver disease.,

To address this lack of dilatation recent studies on intestinal intraluminal devices ${ }^{9,10}$ have been proposed for the treatment of SBS, but currently their translation to clinical practice seems to be limited. Interestingly, the use of different devices as osmotic expanders have been popularized to increase the surface of several tissues such as; skin, ${ }^{11}$ soft tissue, ${ }^{12}$ or hypospadias cripples ${ }^{13}$ and has become the treatment of choice for many congenital and acquired defects ${ }^{14}$; however, this technique has never been used to expand intestinal segments thus far.

In this study, we describe the use of endoluminal osmotic hydrogel to expand an intestinal segment providing subsequent dilated tissue to successfully perform lengthening procedures.

\section{MATERIALS AND METHODS}

\section{Endoluminal osmotic hydrogel expander}

An osmotic device (Osmed ${ }^{\circledR} ; 22 \times 12$ mm, cod. 352-2030) was used to expand a select segment of intestine. This device consisted of hydrogel made by co-polymers based on Methyl methacrylate and N-Vinyl pyrrolidone. The osmotic device absorbs bodily fluid and grows consistently to a predefined form and size. The increased volume of the implant grows 
between 3- and 12-fold, dependent on the product type, leading to an increased soft tissue area. ${ }^{15}$

\section{Animals and anesthesia}

Animal experiments were approved by the Institutional Review Board of the Catholic University of Rome (Prot. Numb.1422/13) and performed in accordance with the EU Directive 2010/63/EU for animal experiments guidelines. To respect the ethical imperative to use the minimum necessary number of animals, power calculation analysis was performed to predict the adequate experimental power and sample number.

A total of five New Zealand rabbits (average weight: 2.52 \pm 0.02 irrespective of gender) were obtained. All the animals had free access to food and water. All surgical procedures and euthanasia were performed under general anesthesia with inhaled oxygen and isoflurane. Pulmonary ventilation after orotracheal intubation was performed.

\section{Surgical procedure}

A 2-cm midline incision was made in the abdomen of each animal. The abdominal cavity was entered, the small bowel was identified, and the intestinal appendix was delivered out of the abdominal cavity. The appendix was divided into two parts, one remained the control, the other had the implant inserted (Figure 1). The proximal and distal ends of the isolated segments were secured with Endo GIA ${ }^{\mathrm{TM}} 30 \mathrm{~mm}$ TriStapler (Covidien, Medtronic Limited; UK) and tied off with 6/0 suture (Prolene, Ethicon Corporation, USA).

The segment with the expander was secured to the parietal peritoneal surface to prevent potential surgical complications such as; volvulus, internal hernia using the technique proposed by Kimura for lengthening. ${ }^{16}$ The abdominal wound was then closed in layers. After 4 weeks the rabbits were euthanized by intravenous injection of $\mathrm{KCl}$ to induce cardiac arrest. Isolated intestinal segments were harvested for the analysis.

The animals were fed standard rodent chow ad libitum. Animal weights and blood tests were performed weekly.

\section{Histology}

Animals were sacrificed 4 weeks postoperatively. The collected full-thickness samples were fixed in zinc-formalin, embedded in paraffin, sectioned and stained with hematoxylin and eosin. The histology of the tissue was investigated for submucosa, muscularis, and serosa according to previous study. ${ }^{17}$ Image J software was used to calculate mucosa surface area. Mucosa surface index analysis was performed according to the formula described by Kisielinski et al. ${ }^{18}$

\section{Analysis}

Results are expressed as the mean \pm SEM. Analysis was performed using the Wilcoxon-test (Prism software; GraphPad Software, Inc., San Diego, CA). A value of $p<0.05$ was considered to be statistically significant.

\section{RESULTS}

Intestinal distraction induced lengthening and dilatation Endoluminal osmotic hydrogel expander (EOHE) was introduced in intestinal segment with measuring $3.68 \pm 0.15 \mathrm{~cm}$ in length and $1.77 \pm 0.12 \mathrm{~cm}$ in diameter with a control segment measuring $3.46 \pm 0.19$ in length and $1.46 \pm 0.03 \mathrm{~cm}$. The statistical analysis showed no difference in the two segments in length $(p=0.43)$ and diameter $(p=0.12)$ at the beginning of the experiment.

All rabbits survived after the surgical procedure until euthanasia. At the time of harvest, the intestinal segment with the endoluminal osmotic expander was still secured to the parietal peritoneum surface [Figure 2(A)] and no devicerelated intestinal obstruction, perforation, volvulus or other surgical complications occurred.

Segments with the osmotic hydrogel lengthened significantly from $4.68 \pm 0.35$ to $9.79 \pm 0.35 \mathrm{~cm}(p<0.01)$ with extensive circumferential dilatation also identified $(p<0.01)$ [Figure 2(B-D)].

The osmotic hydrogel was able to promote an increase in length of $167.8 \pm 35.21 \%$ and an increase in width of $240.5 \pm 24.04 \%$ compared with control segments [Figure 2 (D)] after the study periods. No evidence of ischemia or perforation were found and there were no device related complications.

Blood samples were collected weekly for inflammatory markers and hepatic function. Alanine aminotransferase (ALT), alkaline phosphatase (ALP) [Figure 3(A)], albumin and protein levels slightly reduced during the study period but surprisingly the $\gamma$ GT level showed a not significantly raise (from $4 \pm 0.67$ to $7 \pm 0.90$; normal value U/I 0-14) [Figure 3(B)]. During the study period all the inflammatory markers remained stable or slightly reduced (Figure 3).

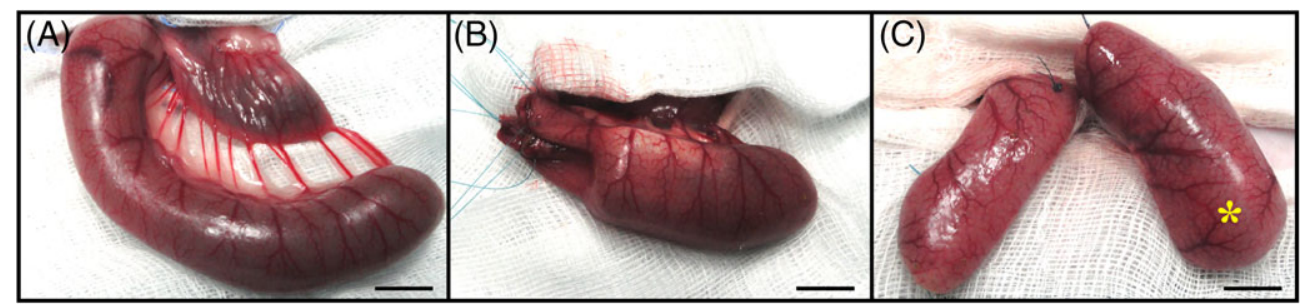

FIGURE 1. Insertion of endoluminal osmotic hydrogel (Osmed ${ }^{\circledR}$ ). (A) The rabbit appendix was identified and easily delivered. (B) The endoluminal osmotic hydrogel was introduced easily into terminal segment of the appendix and (C) a similar segment was identified. Stay suture was put to as a mark for measurement after the study time. Asterisk identified segment with the hydrogel. Scale bars $=1 \mathrm{~cm}$. 


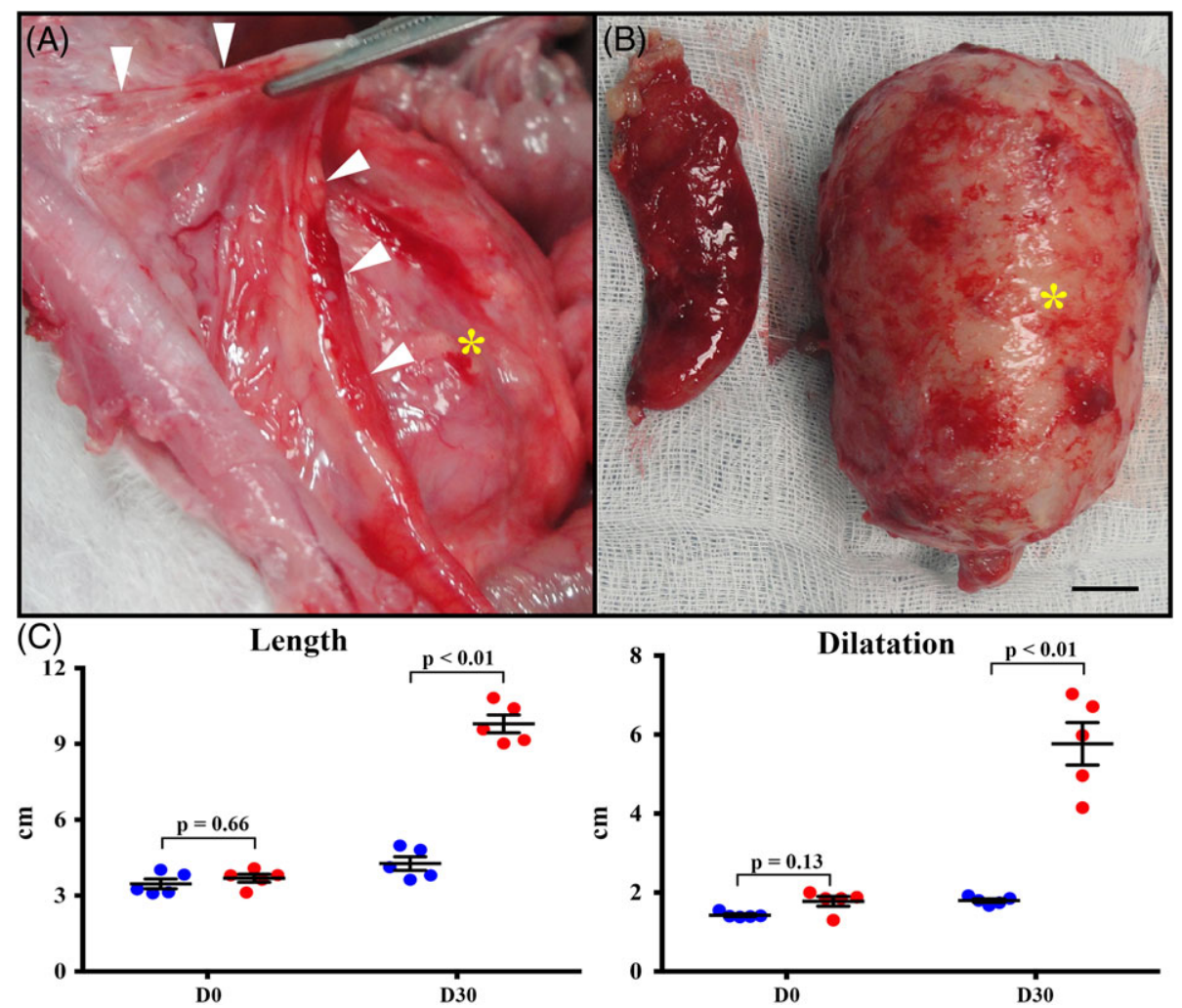

FIGURE 2. Effects of endoluminal osmotic hydrogel expansion. (A) After 4 weeks a vascularized tissue (white arrowheads) is recognized between the antemesenteric surface of the segment with the device (yellow asterisk) and the peritoneal surface. (B) After harvesting of the segments, an extensive distraction of the specimen with the endoluminal osmotic hydrogel (yellow asterisk) was reported. Scale bar $1 \mathrm{~cm}$. (C) Statistical analysis demonstrated that the endoluminal osmotic hydrogel is able to increase significantly the length $(p<0.01)$ and (D) diameter $(p<0.01)$ of intestinal segments. Blue circle: control. Red circle: specimens with endoluminal osmotic hydrogel.

\section{Body weight changes}

Throughout the study, all the animals remained healthy presenting a slight increase in percentage of body weight of $12.6 \pm 3.18 \%$ (from: $2.52 \pm 0.02$ to $2.84 \pm 0.08 \mathrm{~kg} ; p=.06$ ).

\section{Histologic changes}

Hematoxylin and eosin staining revealed conservation of the overall intestinal architecture in both groups but muscular hypertrophy and flattening of the pseudo-stratified epithelium was identified. The osmotic hydrogel creates a significant reduction ( $p=0.03$ ) of the mucosal surface from $7.55 \pm$ 0.96 to $3.84 \pm 0.68 \mu \mathrm{m}^{2}$. The mucosa surface index of the non-expanded segment $0.015 \pm 0.860$ compared with the index from the expanded segment $0.019 \pm 0.329$ showed no difference.

These data suggest that the mechanical expansion from the osmotic hydrogel reduced the mucosa surface without altering the mucosa surface index implying that this technique may only create a temporary modification and not a full structural alteration of the villi-crypt morphology (Figure 4).

\section{DISCUSSION}

In this study, we hypothesized that using a self-inflating hydrogel would lead to continuous bowel stretching of the isolated small intestine. We aimed to test the possibility to expand a select segment of bowel in a short period of time using a well-defined osmotic expander.

Controlled tissue expansion is a technique already used in the clinical scenario for the treatment of SBS. Bianchi introduced the concept of controlled tissue expansion (CTE) modifying the nipple valve idea of Georgeson ${ }^{19}$ and proposed this approach serially within the intestinal rehabilitation program held in Manchester. ${ }^{20}$ Using the clamping recycling approach of tube jejunostomy, this technique allows controlled expansion of the bowel. CTE has been reported to cause bowel dilatation in 20/24 weeks. $^{21}$ The additional absorptive tissue, which is progressively generated, is essential for autologous reconstruction procedures, restoration of gastrointestinal propulsion, epithelial absorption of nutrients and to reduce morbidity. ${ }^{2}$ Disadvantages can be irritation of the skin due to leaking around the tube, multiple extrusions of the tube from the abdomen and the potential for patient discomfort and pain.

The possibility to lengthen intestinal segments using distraction-induced devices seems to be a useful method with potential advantages for the treatment of SBS. Recently, a variety of devices and operative approaches have been tested in animals for the treatment of SBS, but none of these approaches have been translated into clinical practice thus far. Self-expanding, shape-memory polymer cylinder, ${ }^{9}$ catheter 
(A)

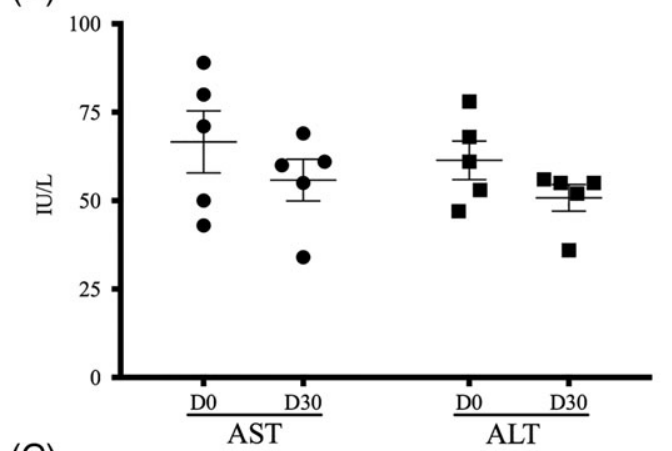

(C) 7.5
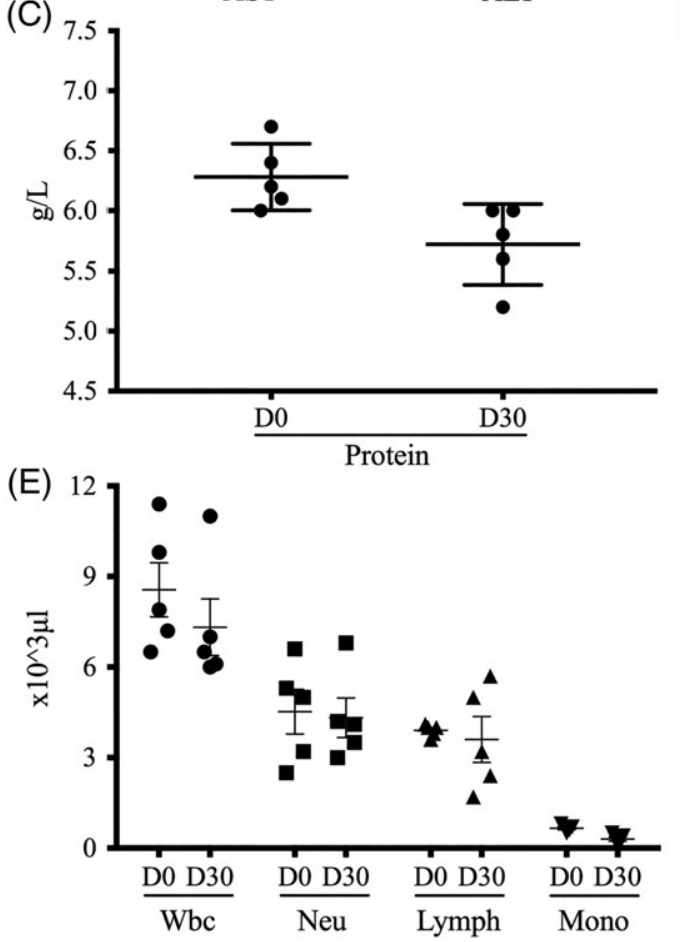

(B)

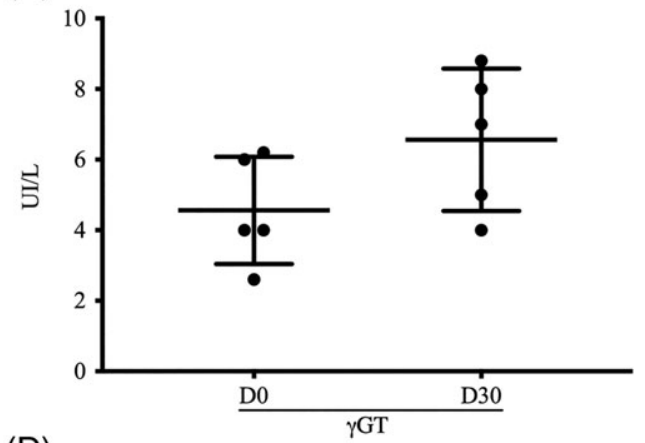

(D)

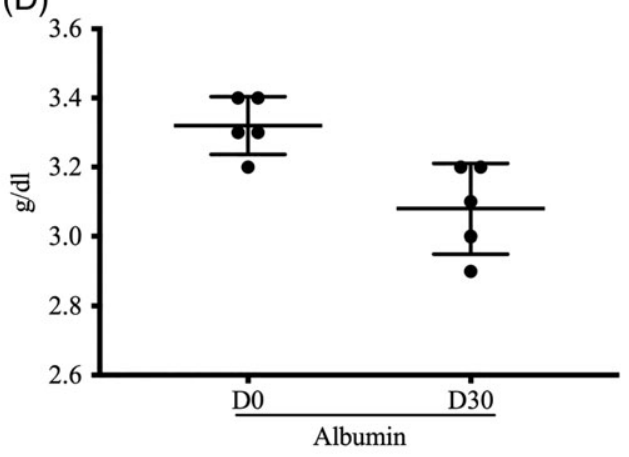

FIGURE 3. Blood test. (A-D) Hepatic markers and (D) inflammatory markers reveal no alterations of the liver function and no infections due to the endoluminal osmotic hydrogel. Normal level of markers: AST (UI/L 34-129); ALT (UI/L 48-80); $\gamma$ GT (UI/L 0-14); Prot (g/L 5,4 - 8,3); ALB (Albumine) (g/dL 2.4-4.6); WBC ( $\left.\times 10^{3} \mu \mathrm{L} 6.3-10.0\right)$; Neu ( $\left.\times 10^{3} \mu \mathrm{L} 1.4-3.2\right)$; Lymph ( $\left.\times 10^{3} \mu \mathrm{L} 30.3-7.0\right)$; Mono $\left(\times 10^{3} \mu \mathrm{L} 0.05-0.45\right)$.

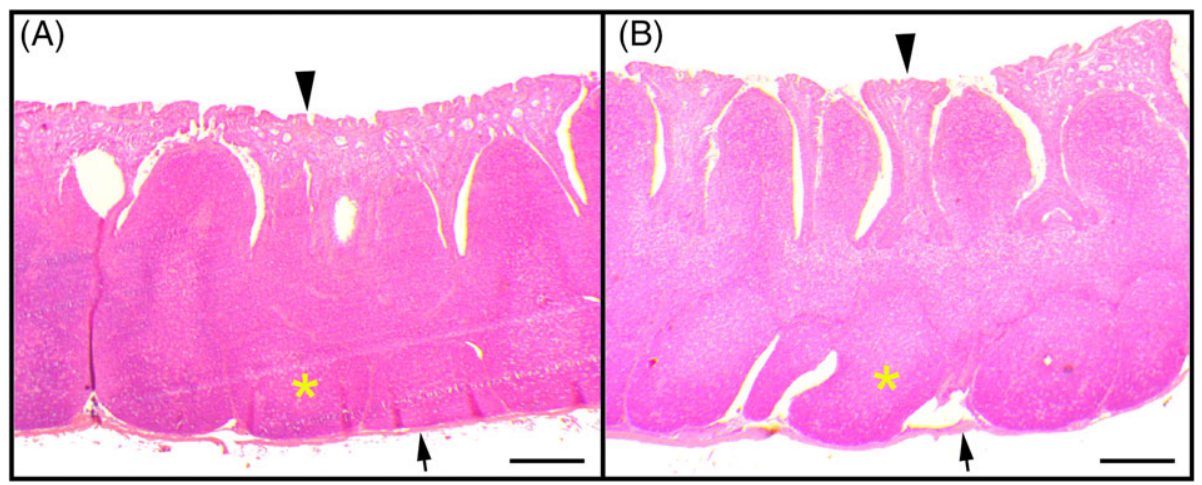

FIGURE 4. Representative bowel from EOHE experiment stained with Hematoxylin and Eosin. In control (A), normal histological intestinal morphology is evident. (B) An example of bowel that underwent tissue expansion. The structure of the mucosal layer exposed to the tissue expander remained intact without ulceration (black arrow head). Yellow asterisk shows lymphatic tissue of the appendix. The villi in the treated bowel are flatter when compared with the control while the muscular layer appears to be thicker (black arrow). Scale bars $=100 \mu \mathrm{m}$. 
device $^{22}$ and a telescopic hydraulic device ${ }^{10}$ have all been tested, but their translations seem to be limited by the need for complex activation mechanisms or the surgical risks associated. These devices appeared challenging to use in a clinical scenario because of reported discomfort, pain and complications. $^{21}$

In this experimental study we aimed to test the ability to expand a select segment of bowel using a well-defined osmotic hydrogel (Osmed ${ }^{\circledR}$ ), already in use in different tissues but never in the intestine. The self-inflating tissue expander used in our study allows osmotic movement of tissue fluid into the implant, which then increases in volume while maintaining its shape. The cylinder used in our study can expand up to five times its original volume (3-30 mL).

Osmed $^{\circledR}$ hydrogel has been previously reported to distract tissue safely for ophthalmic, ${ }^{23,24}$ dental $^{25}$ and plastic surgery. $^{26}$ Interestingly, Osmed ${ }^{\circledR}$ has also been tested in pediatric population for hypospadias repair, in which a selfinflating cylindrical expander was used to allow excision of scar tissue and the potential to cover with expanded native skin. ${ }^{14,27}$

In this in vivo research, an advantage of hydrogel is that it can perform a slow, progressive and continuous expansion over the intestinal anatomical structures. This characteristic is important because fast expansion can damage the villuscrypt. $^{28}$ The self-inflating hydrogel used in our study is mainly formed by water therefore, it permits a high degree of flexibility and may reduce irritation of the surrounding tissues. ${ }^{29}$

Interestingly, our experiment showed not only a dramatic increase in length and diameter in the intestinal segment caused by the osmotic hydrogel but also a revascularization in the antemesenteric border of the isolated bowel segment.

In a previous studies, ${ }^{30,31,32,33}$ structural and histological changes have been found in specimens dilated with osmotic hydrogel, suggesting a similar mechanical stress on the epithelial surface. Hypothetically, shortening the time of distraction from 4 to 3 weeks may represent a possible solution and further experimental work will be needed to explore this hypothesis. The regeneration of epithelium after compression should not be a major issue due to the high regenerative capacity of the intestinal stem cells. $^{34}$ The inflammatory markers remained within the normal range. Analyzing the hepatic function profile, only the $\gamma \mathrm{GT}$ level increased within the normal range (from $4 \pm 0.67$ to $7 \pm$ 0.90 ; normal value U/I 0-14) but no macroscopic hepatic anomalies were identified during retrieval surgery. At present, we do not have an explanation for this phenomenon but will be monitored in further studies.

A limitation of this study was the necessity to use the functional appendix of the rabbit. The ready-made osmotic hydrogel was too big to be inserted into a jejunal segment whilst the appendix had adequate dimensions to insert the Osmed $^{\circledR}$ device endoluminally. Further studies will be needed to test this osmotic expander in larger animal with a more adequate caliber of small bowel.

In conclusion, the use of distraction-induced enterogenesis with an osmotic hydrogel, like Osmed ${ }^{\circledR}$, can lead to a significant increase of length and circumferential diameter of the bowel over a short period of time. This technique has the potential to avoid the formation of stomas with subsequent advantages to the patients and their families. Potentially this hydrogel may provide a new method of controlled tissue expansion for the treatment of patients with short bowel syndrome.

\section{AUTHOR CONTRIBUTIONS}

R.C. designed the study, interpreted distraction enterogenesis results and wrote the body of the article.

R.C. and C.O. performed the surgical procedures on the animals.

G.P. performed a literature review.

V.S., A.I., and A.M. supervised the study and critically appraised the article.

A.M. obtained funding for the study.

All authors approved the final manuscript.

\section{ACKNOWLEDGMENTS}

Three native English-speaking people (Dr Gillham J, Miss Richardson J, Mr Roberts N.) have kindly critically read and approved the written English of this article.

\section{REFERENCES}

1. Coletta $R$, Khalil BA, Morabito A. Short bowel syndrome in children: surgical and medical perspectives. Semin Pediatr Surg 2014;23(5): 291-297.

2. Bianchi A, Morabito A. The dilated bowel: a liability and an asset. Semin Pediatr Surg 2009;18(4):249-257.

3. Bianchi A. From the cradle to enteral autonomy: the role of autologous gastrointestinal reconstruction. Gastroenterology 2006;130(2 Suppl 1):S138-S146.

4. Sanchez SE, Javid PJ, Healey PJ, Reyes J, Horslen SP. Ultrashort bowel syndrome in children. J Pediatr Gastroenterol Nutr 2013; 56(1):36-39.

5. Bakker $H$, Bozzetti $F$, Staun M, Leon-Sanz M, Hebuterne $X$, Pertkiewicz M, Shaffer J, Thul P. Home parenteral nutrition in adults: a european multicentre survey in 1997. ESPEN-home artificial nutrition working group. Clin Nutr 1999;18(3):135-140.

6. Beath S, Pironi L, Gabe S, Horslen S, Sudan D, Mazeriegos G, Steiger E, Goulet O, Fryer J. Collaborative strategies to reduce mortality and morbidity in patients with chronic intestinal failure including those who are referred for small bowel transplantation. Transplantation 2008;85(10):1378-1384.

7. Cohran V. Intestinal failure: the long and short of the matter. J Pediatr 2015;167(1):6-8.

8. Kelly DA. Intestinal failure-associated liver disease: what do we know today? Gastroenterology 2006;130(2 Suppl 1):S70-S77.

9. Fisher JG, Sparks EA, Khan FA, Dionigi B, Wu H, Brazzo J 3rd, Fauza D, Modi B, Safranski DL, Jaksic T. Extraluminal distraction enterogenesis using shape-memory polymer. J Pediatr Surg 2015; 50(6):938-942.

10. Demehri FR, Utter B, Freeman JJ, Fukatsu Y, Luntz J, Brei D, Teitelbaum DH. Development of an endoluminal intestinal attachment for a clinically applicable distraction enterogenesis device. J Pediatr Surg 2016;51(1):101-106.

11. Lohana P, Moiemen NS, Wilson YT. The use of Osmed(TM) tissue expanders in paediatric burns reconstruction. Ann Burns Fire Disasters 2012;25(1):38-42.

12. Uijlenbroek HJ, Liu $Y$, He JF, Visscher $C$, van Waas MA, Wismeyer D. Expanding soft tissue with Osmed tissue expanders in the goat maxilla. Clin Oral Implants Res 2011;22(1):121-128.

13. Lansdale N, Henderson L, Hennayake S. Novel use of an osmotic self-inflating tissue expander for hypospadias revision surgery. Urology 2015;85(4):924-926. 
14. Gronovich $\mathrm{Y}$, Binenboym $\mathrm{R}$, Retchkiman $\mathrm{M}$, Eizenman N, Lotan $\mathrm{A}$, Stuchiner B, Tuchman I. Reconstruction in plastic surgery using osmotic tissue expanders. Harefuah 2015;154(3):155-158. 213.

15. Anwander T, Schneider M, Gloger W, Reich RH, Appel T, Martini M, Wenghoefer M, Merkx M, Berge S. Investigation of the expansion properties of osmotic expanders with and without silicone shell in animals. Plast Reconstr Surg 2007;120(3):590-595.

16. Kimura K, Soper RT. A new bowel elongation technique for the short-bowel syndrome using the isolated bowel segment lowa models. J Pediatr Surg 1993;28(6):792-794.

17. Miyasaka EA, Okawada M, Utter B, Mustafa-Maria H, Luntz J, Brei D, Teitelbaum DH. Application of distractive forces to the small intestine: defining safe limits. J Surg Res 2010;163(2):169-175.

18. Kisielinski K, Willis S, Prescher A, Klosterhalfen B, Schumpelick V. A simple new method to calculate small intestine absorptive surface in the rat. Clin Exp Med 2002;2(3):131-135.

19. Georgeson K, Halpin D, Figueroa R, Vincente $Y$, Hardin W, Jr. Sequential intestinal lengthening procedures for refractory short bowel syndrome. J Pediatr Surg 1994;29(2):316-20; discussion 320-1.

20. Khalil BA, Ba'ath ME, Aziz A, Forsythe L, Gozzini S, Murphy F, Carlson G, Bianchi A, Morabito A. Intestinal rehabilitation and bowel reconstructive surgery: improved outcomes in children with short bowel syndrome. J Pediatr Gastroenterol Nutr 2012;54(4):505-509.

21. Murphy F, Khalil BA, Gozzini S, King B, Bianchi A, Morabito A. Controlled tissue expansion in the initial management of the short bowel state. World J Surg 2011;35(5):1142-1145.

22. Demehri FR, Freeman JJ, Fukatsu Y, Luntz J, Teitelbaum DH. Development of an endoluminal intestinal lengthening device using a geometric intestinal attachment approach. Surgery 2015;158(3): 802-811.

23. Hou Z, Yang Q, Chen T, Hao L, Li Y, Li D. The use of self-inflating hydrogel expanders in pediatric patients with congenital microphthalmia in China. J AAPOS 2012;16(5):458-463.
24. Sivardeen A, Laughton D, Wolffsohn JS. Randomized crossover trial of silicone hydrogel Presbyopic contact lenses. Optom Vis Sci 2016;93(2):141-149.

25. Karner C, Wharton KA Jr, Carroll TJ. Planar cell polarity and vertebrate organogenesis. Semin Cell Dev Biol 2006;17(2):194-203.

26. Al Madani JO. Second generation self-inflating tissue expanders: a two-year experience. Plast Surg Int 2014;2014:457205.

27. Gronovich Y, Tuchman I, Binenboym R, Eizenman N, Golan J. Reconstruction with an osmotic tissue expander in pediatric patients. Plast Reconstr Surg 2012;129(5):863e-865e.

28. Wiese KG, Heinemann DE, Ostermeier D, Peters JH. Biomaterial properties and biocompatibility in cell culture of a novel selfinflating hydrogel tissue expander. J Biomed Mater Res 2001;54(2): 179-188.

29. Gupta P, Vermani K, Garg S. Hydrogels: from controlled release to pH-responsive drug delivery. Drug Discov Today 2002;7(10): 569-579.

30. Yoshino H, Kimura K, Yamazato M, Scott DH, Soper RT. The isolated bowel segment (lowa model II): absorption studies for glucose and leucine. J Pediatr Surg 1991;26(12):1372-1375.

31. Yamazato M, Kimura K, Yoshino H, Murr M, Ellsbury D, Soper RT. The isolated bowel segment (lowa model II): motility across the anastomosis with or without mesenteric division. J Pediatr Surg 1992;27(6):691-695.

32. Bishop WP, Kim SI, Yamazato M, Yoshino H, Kimura K. Mucosal morphology in isolated bowel segments: importance of exposure to luminal contents. J Pediatr Surg 1992;27(8):1061-1065.

33. Demehri FR, Wong PM, Freeman JJ, Fukatsu Y, Teitelbaum DH. A novel double-balloon catheter device for fully endoluminal intestinal lengthening. Pediatr Surg Int 2014;30(12):1223-1229.

34. Okamoto R, Watanabe M. Molecular and clinical basis for the regeneration of human gastrointestinal epithelia. J Gastroenterol 2004;39(1):1-6. 